\title{
THE EXISTENCE OF ALGEBRAIC PLANE CURVES*
}

\author{
BY T. R. HOLLCROFT
}

\section{Curves in General}

1. Introduction. In this discussion, only proper or irreducible algebraic plane curves are to be dealt with; so, unless otherwise stated, the term "curve" will mean an irreducible, algebraic plane curve. The treatment will be from the point of view of algebraic geometry. General curves will be treated first, followed by a discussion of regular and irregular curves. Citations are given by number referring to a brief alphabetical bibliography at the end of the paper. All results that have been previously published are accompanied by a citation.

A curve is of order $n$, class $m$, genus $p$, with $\delta$ nodes, $\kappa$ cusps, $\tau$ bitangents, $\iota$ statangents (contraction of "stationary tangents"). Julius Plücker [15], just a century ago, discovered a very remarkable set of relations involving the above seven characteristics. These relations, which may be expressed in various ways, are called "Plücker's equations." When any three characteristics are given, the other four are uniquely determined by these relations. A curve system, therefore, will be defined by given values of three characteristics. B. Segre [16] has chosen the three characteristics $n, p, \kappa$ and represents a curve system by the symbol $(n, p, \kappa)$. This notation will be used here.

The Plücker relations defining $m, \delta, \iota, \tau$, in terms of $n, p, \kappa$ are

$$
\begin{aligned}
m & =2(n+p-1)-\kappa, \\
\delta & =\frac{1}{2}(n-1)(n-2)-p-\kappa, \\
\iota & =3(n+2 p-2)-2 \kappa, \\
\tau & =\frac{1}{2}\left[(2 n+2 p-\kappa)^{2}-4(5 n+7 p-6)+11 \kappa\right] .
\end{aligned}
$$

A curve system $(n, p, \kappa)$ includes all curves with the given set of characteristics without regard to existence. Other systems may be obtained from a given system $(n, p, \kappa)$ algebraically, that is, $\alpha$ nodes may be added by decreasing $p$ by $\alpha$; $\alpha$ cusps added by simultaneously increasing $\kappa$ by $\alpha$ and decreasing $p$ by

* An address delivered before the Society on February 20, 1937 in New York, by invitation of the Program Committee. 
$\alpha ; \alpha$ nodes changed to cusps or $\alpha$ cusps to nodes by respectively increasing or decreasing $\kappa$ by $\alpha$. These statements hold for positive or negative values of $\alpha$ so that the term "added" implies algebraic addition.

A complete curve system (B. Segre [16], p. 32) is a system that is not contained in a larger system of curves with the same characteristics. A continuous system is one whose descriptive properties only are defined.

A curve system $(n, p, \kappa)$ will be said to exist, that is, to define the characteristics of a curve that exists, when

(a) The algebraic equation of a curve of this system can be written in the polynomial form or in parametric form with one or more parameters and it can be proved that its polynomial equation cannot be factored, that is, is irreducible.

(b) A curve of this system can be constructed and proved to have an irreducible algebraic equation.

(c) A curve of this system can be transformed into a curve known to exist by any non-degenerate rational algebraic transformation.

Conditions (b) and (c) are really contained in (a) so that the question of the existence of a system $(n, p, \kappa)$ becomes-does the curve system define a curve which has an irreducible algebraic equation?

The problem of the existence of plane curves is of basic importance because the existence of curves and manifolds of higher space may be made to depend upon that of plane curves.

In the plane itself, the only noteworthy progress made has been in the case of curves whose only singularities are distinct nodes and cusps. Snyder [20] in 1908 and Severi [19] in 1921 proved that curves with no cusps and any number of nodes up to and including the maximum exist. For curves with only nodes and cusps, the problem of existence is closely related to that of determining the maximum number of cusps of a curve.

2. Conditions for Existence. Conditions for the existence of a curve system $(n, p, \kappa)$ are necessary, sufficient, or both necessary and sufficient.

(1) Necessary conditions. The most obvious necessary conditions for the existence of curves are Plücker's equations. From these relations are derived formulas for the maximum number 
of cusps (Clebsch [2]; Lefschetz [13]; Hollcroft [8]) of a curve of given order and genus. Such limits, called Plücker limits, are necessary conditions.

Cramer [4] in 1750 found certain necessary conditions for the existence of curves with multiple points from the maximum number of intersections of such a curve with another curve through these multiple points. He made a table of the possible multiple points for curves of order $n \leqq 8$. Using a similar process, Plücker $([15]$, p. 215) derived the well known necessary condition $\delta+\kappa \leqq \frac{1}{2}(n-1)(n-2)$.

If the reality of the singularities of the curve is taken into consideration, Klein's Theorem [12], $n+i+2 t^{\prime \prime}=m+k+2 d^{\prime \prime}$, wherein $(i)[k]$ is the number of real (statangents) [cusps] and $\left(t^{\prime \prime}\right)\left[d^{\prime \prime}\right]$ the number of isolated (bitangents) [nodes], is an additional necessary condition for the existence of the curve. The limits to the number of real cusps (Hollcroft [10], pp. 777, 781) for a curve of given order and genus, since they are derived from this theorem and Plücker's equations, are, therefore, necessary conditions.

(2) Sufficient conditions. There are few sufficient conditions for existence that are not also necessary. Sufficient conditions only, however, are given by the two following theorems due to B. Segre ([16], pp. 36-37):

TheOREM $1^{0}$. If a continuous regular system $(n, p, \kappa)$ exists, there exist also the regular systems $\left(n, p_{1}, \kappa_{1}\right)$ where $p_{1}$ and $\kappa_{1}$ satisfy the inequalities $0 \leqq \kappa_{1} \leqq \kappa, p \leqq p_{1} \leqq \frac{1}{2}(n-1)(n-2)-\kappa_{1}$.

THEOREM $2^{0}$. Given two regular systems $\left(n_{1}, p_{1}, \kappa_{1}\right)$ and $\left(n_{2}, p_{2}, \kappa_{2}\right)$, if there exists a curve of one system that touches a curve of the other in $h$ simple points, and the additional intersections $n_{1} n_{2}-2 h$ are distinct points, then there exists a regular system $(n, p, \kappa)$ such that

$$
n=n_{1}+n_{2}, \quad p=p_{1}+p_{2}+h-1, \quad \kappa=\kappa_{1}+\kappa_{2}+h .
$$

Because of the paucity of sufficient conditions for the existence of curves, these theorems are of great importance. The latter theorem has given added significance to the problem of determining the maximum number of contacts of two curves of given systems. 
(3) Necessary and sufficient conditions. Conditions that are both necessary and sufficient for the existence of curves are implied in the definition of existence in the preceding section, namely, that an irreducible polynomial equation of the curve can be either obtained or proved to exist. The fact that the equation is algebraic, rational, integral, and irreducible is sufficient for the curve to satisfy all necessary conditions.

3. The Dimension of a Curve System. The equation of the general curve of order $n$ contains $\frac{1}{2} n(n+3)$ independent coefficients. The curves of this system are in $(1,1)$ correspondence with the points of $S_{n(n+3) / 2}$ and the system is said to be of dimension $n(n+3) / 2$. The values of a certain number of coefficients may be determined in terms of the others by algebraic relations associated with geometric conditions on the curve. Such a condition is expressed by a system of $\alpha \geqq 1$ algebraic equations in the coefficients. The $\frac{1}{2}(n+1)(n+2)$ homogeneous coefficients of the general system of curves may be considered the homogeneous coordinates in $S_{n(n+3) / 2}$. The system of $\alpha$ independent equations in the coefficients that are associated with a certain condition thus define uniquely an algebraic manifold in $S_{n(n+3) / 2}$ whose dimension is $n(n+3) / 2-\alpha$ and whose order is the product of the degrees of the $\alpha$ equations of the system. (Severi [19]).

Conditions involving relations among the coefficients of a curve are of two kinds; first, metric conditions, that is, those associated with position in the plane. Metric relations may be linear or of any order in the coefficients. The second kind of condition is that defining a property of the curve which is invariant under a plane collineation. The associated algebraic relations among the coefficients are called invariants of the curve. The order of an invariant is always greater than unity. Lefschetz ([13], p. 24) has proved that a curve of order $n$ and genus $p \geqq 2$ cannot have more than $\frac{1}{2} n(n+3)-8$ independent invariants.

With each node of a curve is associated one invariant and with each cusp, two invariants. It has been proved (Severi [19]; Snyder [20]) that the $\delta$ nodal invariants associated with any number $\delta \leqq \frac{1}{2}(n-1)(n-2)$ of distinct nodes of a curve of order $n$ are independent. It is also true that the cuspidal invariants are all independent for curves of low order that have been treated in 
detail algebraically. Likewise, from Plücker's equations there results the necessary relation

$$
d_{0}^{\prime}=\frac{1}{2} n(n+3)-\delta-2 \kappa=\frac{1}{2} m(m+3)-\tau-2 \iota,
$$

which (for the curve system whose only singularities are distinct nodes and cusps) appears to express the well known fact that the number of independent coefficients in the equation of a curve in point coordinates equals the number in the equation of the same curve in line coordinates.

In 1913, Lefschetz ([13], p. 26) stated his postulate of singularities which is to the effect that each distinct cusp of a curve is always associated with two independent invariants. This postulate is a statement of what had been found true in all cases investigated up to that time. It could not be proved true in all cases, but the above relation for $d_{0}^{\prime}$ seemed to indicate its validity.

In 1929, B. Segre ([16], pp. 34-35; [17]) discovered a curve system with distinct cusps, to be discussed in section 8 , that does not satisfy the Lefschetz postulate. This contradiction is sufficient to prove that the postulate does not hold in all cases.

With B. Segre [16], the first virtual dimension of the system $(n, p, \kappa)$ is $d_{0}^{\prime}=3 n+p-\kappa-1$. The virtual dimension $d_{0}$ of a curve system with given singularities (not defined in position) is the number of independent coefficients that would remain in the equation of a curve of this system if all of the singularity invariants were considered independent. The effective dimension $d$ is the actual number of independent coefficients in the equation of such a curve system. For a curve system whose only singularities are distinct nodes and cusps, $d_{0}^{\prime}=d_{0}$.

The effective dimension $d$ of an irreducible curve system is also defined as the dimension, reduced by one, of the characteristic series of the curve. The characteristic series has an index of specialty $i \geqq 0$ and a deficiency $\omega \geqq 0$. By the Riemann-Roch Theorem, $d+\omega=d_{0}+i$. B. Segre $([16]$, p. 33) has proved that $d \geqq d_{0}$ for all complete, continuous curve systems. He calls a curve system regular for which $d=d_{0}$ and irregular for which $d>d_{0}$.

Although proved not to apply in all cases, the Lefschetz postulate can now be partially revived, that is, all regular curves satisfy the Lefschetz postulate. The converse, however, is 
not true since other than cuspidal invariants may be dependent (see $\$ 10)$.

4. Curves with Distinct Nodes and Cusps. In 1913, Lefschetz [13] derived from Plücker's equations the following limits to the number of cusps of a curve of given order and genus:

For

$$
\begin{aligned}
0 & \leqq p \leqq p_{0}, \\
\kappa & \leqq \frac{3}{2}(n+2 p-2) ; \\
p_{0}<p & \leqq \frac{1}{6}(n-2)(n-3), \\
\kappa & \leqq 2(n+p)-\frac{1}{2}\left\{11+(24 p-8 n+25)^{1 / 2}\right\} ; \\
\frac{1}{6}(n-2)(n-3) & \leqq p \leqq \frac{1}{2}(n-1)(n-2), \\
\kappa & =\frac{1}{2}(n-1)(n-2)-p .
\end{aligned}
$$

In the above formulas, $p_{0}=\left[\frac{1}{2}\left\{n+1-(4 n+1)^{1 / 2}\right\}\right]$. The genus $p_{0}$ is the least genus of the curve systems of minimum class for a given $n$

The Plücker limits for $p \leqq p_{0}$ and $p>p_{0}$ result from the inequalities $\iota \geqq 0$ and $\tau \geqq 0$ respectively, that is, the fact that none of Plücker's numbers can be negative when the curve is irreducible. Curves with the maximum number of cusps for a given order and genus have been designated maximal cuspidal curves (Hollcroft [9]).

Lefschetz [13] and Coolidge [3] have established the existence of maximal cuspidal curves of genus $p \leqq p_{0}$ and by Segre's Theorem $1^{\circ}$ ([16], p. 36), the existence of all curves with distinct nodes and cusps of genus $p \leqq p_{0}$ is proved. All such curves are also regular $([16]$, p. 33$)$.

If a curve exists, its reciprocal exists. The reciprocals of curves of order $n$ and genus $p \leqq p_{0}$ when $n-2 p+2<\kappa \leqq \frac{3}{2}(n+2 p-2)$ are curves of order $m$ for which it may be that $p>\left[\frac{1}{2}\{m+1\right.$ $\left.-(4 m+1)^{1 / 2}\right\}$ ]. In such cases, however, the number of cusps $\iota$ of the reciprocal is always less than $m+2 p-2$. The existence of these curves of genus greater than $p_{0}$ does not aid in establishing the existence of curves of genus greater than $p_{0}$ with a number of cusps equal to or near the Plücker limit for that genus. With the exception of an irregular system shown to exist in $\S 8$, no general system of maximal cuspidal curves of genus $p>p_{0}$ has been proved to exist.

The two following theorems define curve systems whose reciprocals are maximal cuspidal curve systems, and conversely: 
1. Every curve system of order $n$ for which $\kappa \leqq 1$ and $\delta \geqq n-4$ is the reciprocal of a maximal cuspidal curve system of genus $p \leqq p_{0}$.

2. Every curve system of order $n$ and class $m$ with $\kappa \geqq 0$ cusps and $\delta \leqq n-5$ nodes is the reciprocal of a maximal cuspidal curve system of order $m$ and genus $p$ such that $p_{0}<p \leqq(m-2)(m-3) / 6$.

\section{Regular Curve Systems}

5. B. Segre's Results. By establishing the completeness of the characteristic series* of a complete continuous system of curves $(n, p, \kappa)$ for which $\kappa<3 n$ and $n$ is sufficiently large, $\dagger \mathrm{B}$. Segre ([16], p. 33) proved:

All continuous systems of irreducible algebraic curves whose only singularities are distinct nodes and cusps, provided that $\kappa<3 n$, are regular, that is for such a system, $d_{0}^{\prime}=d_{0}=d=3 n$ $+p-1-\kappa$.

In the same paper $([16]$, pp. 36-37), he gave the two Theorems $1^{0}$ and $2^{0}$, which have been stated as sufficient conditions for existence in $\$ 2$. A third theorem, Theorem $3^{0}$ ([16], p. 37) is derived from Theorem $2^{0}$ by considering the second curve system as a line counted twice, that is, $\left(n_{2}, p_{2}, \kappa_{2}\right)$ $\equiv(2,-1,1)$.

TheOREM $3^{0}$. If a regular system $\left(n_{1}, p_{1}, \kappa_{1}\right)$ exists for $n_{1} \geqq 3$, there exists also the regular system $(n, p, \kappa)$ such that

$$
n=n_{1}+2, \quad p=p_{1}+n_{1}-2, \quad \kappa=\kappa_{1}+n_{1}+1 .
$$

From the four preceding theorems, Segre derives the existence theorem:

There exist continuous regular systems $\left(2 n, n^{2}-3 n+1, n^{2}\right)$ for $n \geqq 3$ and $\left(2 n+1, n^{2}-2 n+1, n^{2}+n-1\right)$ for $n \geqq 1$ of irreducible curves with only cusps.

Theorems $1^{0}, 2^{0}, 3^{0}$ are given without proof.

There are questions as to the field of validity of Theorem $3^{0}$

* The proof is similar to that of Severi [19] in the case of nodes. Severi proved that all continuous systems $(n, p, 0)$ are regular.

$\dagger$ By Plücker's equations, the least value of $n$ for which $k=3 n$ is 11 . This is a self dual curve and probably does not exist. For $m \neq n$, when $\kappa=3 n$, $m=n(n-10)$ and $p-1=(m+n) / 2$. The system $(12,19,36)$ is the one of lowest order for $m \neq n$. The least value of $n$ for which this theorem holds when $\kappa$ $=3 n-1$ probably lies between 12 and 17 . 
and therefore of the above existence theorem derived partially from this theorem. This will be discussed in section 7 .

6. Zariski's Results. Zariski studied the surface $z^{n}=f(x, y)$ where $f(x, y)=0$, the branch curve [21] of the surface, is an algebraic curve with given singularities. In 1929, he proved the theorem [22]:

If $n=q^{\alpha}$, where $q$ is a prime number, and $f(x, y)=0$ is an irreducible algebraic curve, then the surface $z^{n}=f(x, y)$ is regular.

A regular surface $F$ of order $n$ is one for which $p_{g}-p_{a}=0$, where $p_{a}$, the arithmetic genus of $F$, is one more than the virtual dimension of the system of adjoint surfaces of order $n-4$ and $p_{g}$, the geometric genus of $F$, is one more than the effective dimension of the same adjoint system. The geometric genus is also defined as the number of everywhere finite abelian double integrals of the first kind associated with the surface $F$. The difference $I=p_{0}-p_{a} \geqq 0$ is called the irregularity of $F$.

Let $f(x, y)=0$ be a curve $f$ of order $n$ with no singularities other than distinct nodes and cusps. The surface $F$ with equation $z^{n}=f(x, y)$ has compound double points at the nodes and cusps of $f$ in the plane $z=0$. An adjoint surface $\phi(x, y, z)=0$ of order $n-4$ does not pass through the ordinary double points of $F$, but may pass through compound double points of $F$.

By studying the behavior, at these double points of $F$, of the abelian integrals $\iint\left[\phi(x, y, z) / z^{n-1}\right] d x d y$, Zariski ([24], p. 488) proved these theorems:

1. The adjoint surfaces of $F$ do not pass through double points of $F$ which are nodes of $f$.

2. At each double point of $F$ which is a cusp of $f$, the adjoint surfaces of $F$ have $[n / 6]$ consecutive basis points $([n / 6]$ is the largest integer contained in the quotient $n / 6)$.

The second result leads to the following limit ([24], p. 500) to the number of cusps of an irreducible curve of order $n$,

$$
\kappa<\frac{1}{2}(n-\beta)(n-\beta-3)+2, \quad \beta=[(n-1) / 6] . *
$$

For a given $n$, this limit defines a smaller number of cusps than the Plücker limit in certain cases $([24]$, p. 501).

* In reference [25], p. 174, the equality sign is also given in this limit. In accordance with the derivation, the equality sign is not admissible. 
7. The Existence of Curves of Genus $p \leqq 5$. Lefschetz ([13], pp. 37-39) investigated the existence of curve systems of low genus with only distinct nodes and cusps and proved that all maximal cuspidal curve systems of genus $p \leqq 5$ exist with the three exceptions $(7,3,11),(8,4,14),(8,5,16)$.

As stated in $\$ 4$, all curves having only distinct nodes and cusps and of genus $p \leqq p_{0}$ exist. In determining the existence of curve systems of genus $p \leqq 5$, therefore, it is only necessary to deal with curve systems for which $p_{0}<5$. The inequality $p_{0}<5$ gives $n \leqq 16$. The reciprocals of all such curves of order $n \geqq 10$ are curves of order $n \leqq 8$ which do not have the maximum number of cusps for that order and genus, so that all exist. For $n=9$, however, among the reciprocals there are two additional systems, $(7,4,11)$ and $(8,5,15)$, which cannot be proved to exist.*

Zariski ([24], p. 501) has shown that the systems $(7,4,11)$ and $(7,3,11)$ do not exist. This contradicts B. Segre's Theorem $3^{0}$. The curve system of order 7 with 11 cusps is the curve of lowest order satisfying the Plücker limits that does not exist. $\dagger$

In a separate paper [23], Zariski gives a direct proof by plane geometry that the system $(8,5,16)$ does not exist. He points out in a footnote in his paper $([23]$, p. 309) that B. Segre has stated ([16], p. 38 , footnote) that the system $(8,5,16)$ exists as a result of the preceding theorems of that paper.

A contradiction between Zariski's limit $\kappa<\frac{1}{2}(n-\beta)(n-\beta-3)$ $+2, \beta=[(n-1) / 6]$, and B. Segre's Theorem $3^{0}$ occurs only in the case of curves of orders 7 and 8 . B. Segre's existence theorem which affirms the existence of regular curve systems $(2 n$, $\left.n^{2}-3 n+1, n^{2}\right), n \geqq 3$, and $\left(2 n+1, n^{2}-2 n+1, n^{2}+n-1\right), n \geqq 1$, coincides with the Plücker limit for $n \leqq 8$ and gives a much smaller limit for $n \geqq 9$. The Zariski limit defines a maximum number of cusps smaller than the Plücker limit only for the six orders $n=7,8,13,14,19,25$. For all orders except 7 and 8 (in which cases B. Segre's existence theorem allows 11 and 16 cusps, Zariski's limit 10 and 15 respectively), the existence state-

* The three exceptional systems given by Lefschetz constitute all of the doubtful self dual maximal cuspidal curve systems for $p \leqq 5$. Of the two additional systems, $(7,4,11)$ is of higher genus than $(n-2)(n-3) / 6$ for $n=7$ and $(8,5,15)$ is "between" the two doubtful systems of order 8 given by Lefschetz.

$\dagger$ The conclusion of Crone $[5]$ that the system $(6,2,7)$ does not exist is not justifiable. 
ment of B. Segre defines a smaller number of cusps than the Zariski limit.

In addition to showing that the curve systems $(7,4,11)$, $(7,3,11)$ and $(8,5,16)$ do not exist, Zariski's theorem ([22], p. 494) limits the positions of cusps as follows on certain curve systems :

$(7,5,10)$, the ten cusps cannot lie on a cubic;

$(8,9,12)$, the twelve cusps cannot lie at the intersections of a cubic and a quartic;

$(8,6,15)$, the 15 cusps cannot lie on a quartic;

$(9,7,21)$, the 21 cusps cannot lie on a quintic.

The first two of these cases are mentioned by Zariski ([24], p. 502). The existence of the last two curve systems is in doubt, but their existence is permitted by the theorem which restricts the positions of their cusps.

The existence of a curve of order 9 with 18 cusps will now be established.

The Cayleyan of an elliptic cubic is a sextic with 9 cusps which has nine contacts with the cubic. Therefore the regular systems $(6,1,9)$ and $(3,1,0)$ contain curves that have 9 contacts. Applying Segre's Theorem $2^{0}$ (see $\$ 2$ ), we find that set $\left(n_{1}, p_{1}, \kappa_{1}\right) \equiv(6,1,9),\left(n_{2}, p_{2}, \kappa_{2}\right) \equiv(3,1,0)$, and $h=9$. The theorem then asserts the existence of the regular curve system $(9,10,18)$.

The reciprocal of $(7,4,11)$ is $(9,4,17)$, which does not exist since $(7,4,11)$ does not exist. In the above paragraph, the system $(9,10,18)$ has been shown to exist, which implies that all curve systems $(9, p, \kappa)$ exist for $p \geqq 10$ and $\kappa \leqq 18$.

This is the first time that the existence has been established of a curve with a number of cusps greater than that possessed by a curve of the same order that does not exist. This adds a new conception to the problem of existence-that distinct nodes added to the singularities of an existing curve system with only distinct nodes and cusps, within the Plücker limits, may give a system that does not exist. In this case, the system $(9,10,17)$ exists. Six nodes added to this system give $(9,4,17)$ whose reciprocal, by Zariski's theorem, does not exist.

It is entirely possible, from the following point of view, for the system $(9,4,17)$ to be non-existent while the system $(9,10,18)$ exists. Since the system $(9,4,17)$ does not exist because (and only because, so far as known proofs are concerned) the 
system $(7,4,11)$ has too many cusps for its order, then, reciprocally, the system $(9,4,17)$ does not exist because it has too many statangents for its class. This does not imply that the system $(9,4,17)$ has too many cusps for its order, but that the combination of 17 cusps and 7 nodes is too much, in that it defines a reciprocal system with too many cusps for its order.

\section{Irregular Curve Systems}

8. The Curve System $f$. The curve system of order $6 \alpha$

$$
f \equiv \phi_{3 \alpha}^{2}+\lambda \psi_{2 \alpha}^{3}=0,
$$

in which $\phi_{3 \alpha}=0, \psi_{2 \alpha}=0$ are two entirely general curve systems of orders $3 \alpha$ and $2 \alpha$ respectively, has been long known, and it has also been known that this system has $6 \alpha^{2}$ cusps at the intersections of $\phi_{3 \alpha}$ and $\psi_{2 \alpha}$. The new property of this system discovered by B. Segre ([16], p. 34) is its irregularity for $\alpha \geqq 3$.

The equation of $f$ contains

$$
\frac{1}{2}(3 \alpha+1)(3 \alpha+2)+(\alpha+1)(2 \alpha+1)-1=\frac{1}{2}(\alpha+1)(13 \alpha+2)
$$

non-homogeneous coefficients. The proof of the irregularity of $f$ consists in showing that all of these coefficients are independent. Segre proves this by showing that the system $f$ is a complete, continuous, irreducible system of curves with a complete characteristic series.

The independence of the coefficients is also proved algebraically. Of the $6 \alpha^{2}$ intersections of $\phi_{3 \alpha}$ and $\psi_{2 \alpha}$, only $6 \alpha^{2}-(2 \alpha-1)(2 \alpha-2) / 2=(\alpha+1)(4 \alpha-1)$ points chosen on $\phi_{2 \alpha}$ count as independent conditions in the determination of $\phi_{3 \alpha}$. Of these $(\alpha+1)(4 \alpha-1)$ points, assume that $\alpha(2 \alpha+3)$ are chosen arbitrarily, thus determining $\psi_{2 \alpha}$, and that the remaining $2 \alpha^{2}-1$ are chosen on $\psi_{2 \alpha}$. Then $\phi_{3 \alpha}$ still contains $3 \alpha(3 \alpha+3) / 2$ $-(\alpha+1)(4 \alpha-1)=\frac{1}{2}(\alpha+1)(\alpha+2)$ arbitrary parameters and therefore $f$ contains $\frac{1}{2}(\alpha+1)(\alpha+2)+1$ arbitrary parameters. The position of a cusp in the plane is determined by two parameters, on a given curve by one parameter. Of the $(\alpha+1)(4 \alpha-1)$ given cusps on $\psi_{2 \alpha}$, only $\alpha(2 \alpha+3)$ positions are independent and account for two parameters each, while the remaining $2 \alpha^{2}-1$ positions on $\psi_{2 \alpha}$ account for one parameter each. Then, so far as determining the positions of $(\alpha+1)(4 \alpha-1)$ cusps is concerned, the number of conditions on $f$ would be $2 \alpha(2 \alpha+3)$ 
$+2 \alpha^{2}-1=6 \alpha^{2}+6 \alpha-1$. If the positions of these $(\alpha+1)(4 \alpha-1)$ cusps are considered as not given, there would be restored to the system $f$, on which they were considered as given, $6 \alpha^{2}+6 \alpha-1$ parameters. It was shown that the system $f$ for which the positions of these cusps were given contained $\frac{1}{2}(\alpha+1)(\alpha+2)+1$ parameters. Therefore a curve system $f$ for which no positions of the $6 \alpha^{2}$ cusps are given contains

$$
\frac{1}{2}(\alpha+1)(\alpha+2)+1+6 \alpha^{2}+6 \alpha-1=\frac{1}{2}(\alpha+1)(13 \alpha+2)
$$

independent parameters.

In the above proof, it was revealed that as to the positions of the $6 \alpha^{2}$ cusps of $f: \alpha(2 \alpha+3)$ are independent and require two parameters each; $2 \alpha^{2}-1$ are on $\psi_{2 \alpha}$ and require one parameter each; $(\alpha-1)(2 \alpha-1)$ are gratuitous, that is, they follow from the above.

The characteristics of $f$ are these: $n=6 \alpha, m=6 \alpha(3 \alpha-1)$, $p=12 \alpha^{2}-9 \alpha+1, \delta=0, \kappa=6 \alpha^{2}, \iota=12 \alpha(5 \alpha-3), \tau=27 \alpha(3 \alpha-2)$ $\cdot\left(2 \alpha^{2}-1\right), \quad d_{0}=3 \alpha(2 \alpha+3), \quad d=(\alpha+1)(13 \alpha+2) / 2, \quad I=d-d_{0}$ $=(\alpha-1)(\alpha-2) / 2$.

As will be seen from the characteristics of $f$, the reciprocal $f^{\prime}$ of $f$ has $\tau=0$ which is a condition, when $p>p_{0}$, for the maximum number of cusps. The reciprocal $f^{\prime}$ is of order $6 \alpha(3 \alpha-1)$ and genus $12 \alpha^{2}-9 \alpha+1$. For this order, the value of $p_{0}$ is always less than the genus of $f^{\prime}$ for all values of $\alpha$. Therefore this curve system $f$ has still another new property-its reciprocal is the first maximal cuspidal curve system of general order known to exist for $p>p_{0}$.

Since the system $f$ has no invariants other than cuspidal invariants and since for $f$ we have $I=(\alpha-1)(\alpha-2) / 2$, it follows that out of the total of $12 \alpha^{2}$ cuspidal invariants, $I$ are dependent. This does not mean, necessarily, that some of the cusps of $f$ are dependent. Since a cusp is the limiting case of a node, the two cuspidal invariants take the place of the nodal invariant. Since all nodal invariants have been proved independent, it is probable that at least one of the invariants associated with any cusp is independent.

Since in the case of a curve with distinct nodes and cusps, $d_{0}=n(n+3) / 2-\delta-2 \kappa=m(m+3) / 2-\tau-2 \iota$, and since the effective dimension $d$ of a curve considered as a locus is equal to that of the same curve considered as an envelope, $I=d-d_{0}$ has the same value for the given curve considered either as a locus or as 
an envelope. Nodal invariants are independent, hence, reciprocally, bitangent invariants of a class curve are independent. Therefore if a curve, considered as a locus, has a certain number $I$ of dependent cuspidal invariants, the same curve considered as an envelope has $I$ dependent statangent invariants.

Therefore the reciprocal of an irregular system of irregularity $I$ is an irregular system of irregularity $I$, and if the original system has $K$ dependent cuspidal invariants, the reciprocal system has $K$ dependent cuspidal invariants.

Hence the reciprocal of $f$, which has been shown to be a maximal cuspidal curve system $f^{\prime}$ of genus $p>p_{0}$, has $(\alpha-1)(\alpha-2) / 2$ dependent cuspidal invariants.

The system $f$ is irregular because its cusps occur at the intersections of curves of lower order and not because of an excessive number of cusps for the order of $f$. It is highly probable that systems of order $6 \alpha$ with $6 \alpha^{2}$ cusps, unrestricted a priori in position, exist. In the discussion below, this is shown to be true in the case $\alpha=1$.

Zariski ([24], p. 503) has proved the following theorem:

If $f(x, y)=0$ is an irreducible curve of order $n$ possessing nodes and cusps only, a necessary and sufficient condition that the surface $z^{n}=f(x, y)$ be irregular is that $n$ be divisible by six and that the system $\left|C_{(5 n-18) / 6}\right|$ of the curves of order $(5 n-18) / 6$ passing through the cusps of the curve $f$ should be superabundant. If these conditions are satisfied, the irregularity of the surface is equal to the superabundance of the above system $\left|C_{(5 n-18) / 6}\right|$.

This theorem requires that all surfaces $z^{n}=f(x, y)$ be regular if $f(x, y)=0$ is an irreducible curve of order $n$ with only distinct nodes and cusps whose order $n$ is not divisible by six. This is much more general than the former theorem which required $n$ to be the power of a prime in order for $z^{n}=f(x, y)$ to be regular.

The system $f(x, y) \equiv \phi_{3 \alpha}^{2}+\lambda \psi_{2 \alpha}^{3}=0$, under this theorem, is the only irreducible system known for which the surface $z^{n}=f(x, y)$ is irregular.

The special case of $f$ for $\alpha=1$ defines a system of sextics $C_{6}$ with six cusps on a conic. This system $C_{6}$ is of dimension $d=d_{0}=15$, the same as the dimension of a sextic $C_{6}^{\prime}$ with six cusps in general position.* Zariski $([21]$, p. 320) has proved

* When the positions of the cusps are given, however, the dimensions of these two sextic curve systems are not equal. The $C_{6}$ with 6 cusps at given 
that $C_{6}$ is the branch curve of a general cubic surface and that the fundamental groups of the two types of sextics are distinct. B. Segre $\left([17]\right.$, p. 559) has shown that a $C_{6}^{\prime}$ exists. The fact that $C_{6}$ and $C_{6}{ }^{\prime}$ belong to distinct systems contradicts a theorem by Albanese ([1], part IV).

The above example gives rise to another discovery in curve theory-that a system of irreducible curves with given characteristics $(n, p, \kappa)$ does not necessarily form one irreducible complete system (Zariski [25], p. 175).

The system $f$ is of the form $\phi_{a}{ }^{2}+\psi_{b}{ }^{3}=0,2 a=3 b$. What are the properties of the system defined by this equation when $2 a \neq 3 b$ ?

In order to study this question, consider the continuous system

$$
f_{2} \equiv \phi_{3 \alpha+1}^{2}+\lambda \psi_{2 \alpha}^{3}=0 .
$$

This system is of order $6 \alpha+2$ and has $2 \alpha(3 \alpha+1)$ cusps at the intersections of $\phi_{3 \alpha+1}$ and $\psi_{2 \alpha}$. All of the $\left(13 \alpha^{2}+21 \alpha+6\right) / 2$ coefficients can be proved independent. Also $d_{0}=6 \alpha^{2}+17 \alpha+5$; hence $d-d_{0}=\frac{1}{2}\left(\alpha^{2}-13 \alpha-4\right)$. For $\alpha \leqq 13$, the system $f_{2}$ is incomplete. For $\alpha \geqq 14$, the system $f_{2}$, if irreducible, is a complete, continuous, irregular system.

The equations $f_{3}$ and $f_{4}$ of systems of orders $6 \alpha+3$ and $6 \alpha+4$ respectively may be written similarly and the systems have similar properties.

The surfaces $z^{6 \alpha+i}=f_{i},(i=2,3,4)$, however, are found to be irregular for all values of $\alpha$ for which the above curve systems would be irregular. Since the orders of these surfaces are not multiples of six, by Zariski's theorem ([24], p. 503), quoted in this section, all of these curve systems must be reducible for the values of $\alpha$ for which they would be irregular.

9. The Plane Sections of Tangent Cones. The sections by a plane $\pi$ of the tangent cones to an algebraic surface of order $\nu$ form a complete, irreducible system of curves of order $\nu(\nu-1)$

points on a conic is an $\infty^{4}$ system and the $C_{6}^{\prime}$, the positions of whose cusps are independently assigned, is an $\infty^{3}$ system.

For any $\alpha$, if the positions of all the $6 \alpha^{2}$ cusps are given, the system $f$ is of dimension $1+(\alpha+1)(\alpha+2) / 2$. If the positions of the $6 \alpha^{2}$ cusps of a curve system of order $6 \alpha, \alpha \geqq 2$, are considered independent of each other, the positions of only $3 \alpha(2 \alpha+3) / 2$ cusps can be chosen arbitrarily and the positions of the remaining $3 \alpha(2 \alpha-3) / 2$ cusps follow from these. 
with $\kappa=\nu(\nu-1)(\nu-2)$ and $\delta=\frac{1}{2} \nu(\nu-1)(\nu-2)(\nu-3)$. These are branch curves of the surface. The parametric equations of such a system may be written $\phi(x, y, z)=0, \partial \phi / \partial z=0$, wherein $z$ is the parameter and $\phi=0$ the equation of the surface. B. Segre [13] has proved that, for this system, $d-d_{0}=(\nu-2)(\nu-3)$ $(\nu-4) / 6$. The system is thus irregular for $\nu \geqq 5$. B. Segre has also shown that the nodes and cusps of this system lie at the intersections of curves of lower order.

10. An Irregular System of Quartic Curves. In the two preceding sections, two well known curve systems have revealed a new property, that of irregularity. In this section, another well known curve system, the quartics with three biflecnodes, will be shown to be irregular.

A rational trinodal quartic that has biflecnodes at two of the nodes must have a biflecnode at the third node. The equation of the tri-biflecnodal quartic may be written in the form

$$
x_{1}^{-2}+x_{2}^{-2}=x_{3}^{-2} . \quad \text { (Hilton [7], p. 285.) }
$$

Making the sides of the coordinate triangle any three lines of the plane and writing the equation in integral form, we see that

$$
q \equiv(b x)^{2}(c x)^{2}+(a x)^{2}(c x)^{2}-(a x)^{2}(b x)^{2}=0,
$$

wherein $(a x) \equiv a_{1} x_{1}+a_{2} x_{2}+a_{3} x_{3}, \cdots$. The equation $q$ contains 7 independent coefficients, that is, for the system $q$, the effective dimension $d$ is equal to 7 . The system $q$ is continuous and complete.

Each node is associated with one invariant. Distinct statangents account for no invariants on an order curve, but if both nodal tangents coincide respectively with two statangents with their points of contact at the node, the singularity is associated with three invariants (Hollcroft [11], p. 265). The system $q$ with three biflecnodes is therefore of virtual dimension $d_{0}=5$.

Therefore $d-d_{0}=2$ and the system $q$ is irregular. The order four is evidently the lowest possible order for a complete, continuous, irregular curve system.

The reciprocal $q^{\prime}$ of $q$ is a rational maximal cuspidal sextic with the six cusps on a conic and so associated in three pairs that each pair has a common cuspidal tangent. These three common tangents form a self conjugate triangle of the conic on which lie the six cusps. 
The equation of $q^{\prime}$ contains the same number of independent constants as that of $q$, that is, $d=7$. The invariant postulation of two cusps with a common tangent is six, two for each cusp and two for the coincident cuspidal tangents. This coincidence implies that four tangents coincide, but since there are two distinct points of contact, the tangent is still a bitangent. The system $q^{\prime}$ has four nodes. For $q^{\prime}$, therefore, $d_{0}=27-18-4=5$. Hence $d-d_{0}=2$.

The most interesting thing about this system $q$ and its reciprocal $q^{\prime}$ is that they exhibit cases of irregular systems in which cuspidal invariants are not the dependent invariants. A property of the reciprocal of the tri-biflecnodal quartic is stated as follows: If a rational maximal cuspidal sextic has two pairs of cusps so situated that the cuspidal tangents of both pairs coincide, the cuspidal tangents of the third pair must coincide. The sextic is considered as having the cusps before these further conditions are applied. Thus in the case of $q^{\prime}$, the two dependent invariants are associated with the coincidence of the third pair of cuspidal tangents. In the case of $q$, the two dependent invariants are associated with the coincidence, respectively, of two statangents with the two nodal tangents at the third node.

In both cases, the dependent invariants are associated with the coincidence of tangents, but not with the coincidence of nodal tangents to form a cusp.

11. Curve Systems with Negative Virtual Dimension. It is unlikely that curves exist with only distinct nodes and cusps for which $d_{0}$ is negative, although this is permitted by Plücker's equations. However, systems with higher singularities may exist for which $d_{0}<0$.

The irregular system $f$ is a special case of a general system of the form $\phi_{b \alpha}^{a}+\lambda \psi_{a \alpha}^{b}=0, a>b$. This system is of order $a b \alpha$ with $a b \alpha^{2}$ compound $b$-fold points, each with $b$ coincident tangents. The curve system is irregular and may be reducible for certain values of $a, b, \alpha$. Its effective dimension is easily found, but the virtual dimension is difficult to obtain in a general case because each multiple point contains latent consecutive multiple points.

If $a=b+1$, the $b$-fold points are simple multiple points with $b$ coincident tangents. The system $f_{b}$ is of order $\alpha b(b+1)$ and has $\alpha^{2} b(b+1)$ simple multiple points of order $b$ at the intersections of $\phi_{\alpha b}=0$ and $\psi_{\alpha(b+1)}=0$. Each $b$-fold point contains 
(penultimately) $b-1$ cusps and $(b-1)(b-2) / 2$ nodes and is associated with $\left(b^{2}+3 b-6\right) / 2$ invariants. Hence

$$
d_{0}=\alpha b(b+1)(6 \alpha-2 \alpha b+3) / 2 .
$$

The coefficients of $f_{b}$ are independent, so that for $f_{b}$

$$
\begin{aligned}
d-d_{0}= & \frac{1}{2} \alpha b[(b+1)(2 \alpha b-6 \alpha-3)+(\alpha b+\alpha+3)] \\
& +\frac{1}{2}(\alpha+1)(\alpha+2) .
\end{aligned}
$$

Setting $d_{0}<0$ and solving for $b$, we see that $d_{0}<0$ for $\alpha=1$, $b \geqq 5$, and for $\alpha \geqq 2, b \geqq 4$.

The curve system of lowest order of this form for which $d_{0}$ is negative is of order $30(\alpha=1, b=5)$ and has the equation

$$
\gamma \equiv \phi_{5}{ }^{6}+\lambda \psi_{6}{ }^{5}=0 \text {. }
$$

The system $\gamma$ has 30 simple 5 -fold points, each with 5 coincident tangents and is of genus 135. Each 5 -fold point is associated with 17 invariants. For $\gamma$, therefore, $d=48, d_{0}=495-510=$ -15 , and $d-d_{0}=48-(-15)=63$.

For $\alpha=2, b=4$, the curve system is

$$
\mu \equiv \phi_{8}^{5}+\lambda \psi_{10}^{4}=0 .
$$

The system $\mu$ is of order 40 and has 80 quadruple points, each with four coincident tangents. For this system, $d=110$, $d_{0}=860-880=-20$ and $d-d_{0}=130$.

It may be that equations of this form do not always represent irreducible systems. The above systems $\gamma$ and $\mu$, however, satisfy the known necessary conditions for existence, possess only distinct, simple multiple points, and have a negative virtual dimension.

An interesting question is raised by systems of this kind, as to whether the $d-d_{0}$ dependent invariants occur entirely among the cuspidal invariants implied in the multiple points (of which each $b$-fold point has $b-1$ ), or entirely among the combination invariants (of which each simple multiple point of order $b$ has $b-2$ ), or among both of these sets of invariants. In the examples of irregular curve systems given in the preceding sections, the dependent invariants have always been associated with the coincidence of tangents, and with the coincidence of nodal tangents to form a cusp except in the case of the irregular system of quartics discussed in the preceding section. 


\section{BIBLIOGRAPHY}

1. Albanese, G., Sui sistemi continui di curve piane algebriche, Pisa, Nistri, 1923.

2. Clebsch, A., Journal für Mathematik, vo!. 64 (1864), p. 51.

3. Coolidge, J. L., On the existence of curves with assigned singularities, this Bulletin, vol. 28 (1922), pp. 451-455.

4. Cramer, G., Introduction d l'Analyse des Lignes Courbes Algébriques, Geneva, 1750, pp. 455-459.

5. Crone, C., Sur une espèce de courbes symmetriques de la sixième classe, Acta Mathematica, vol. 2 (1883), pp. 81-96.

6. Haskell, M. W., The maximum number of cusps of an algebraic plane curve and enumeration of self dual curves, this Bulletin, vol. 23 (1917), pp. 164165.

7. Hilton, H., Plane algebraic curves, Oxford, 1920.

8. Hollcroft, T. R., Singularities of curves of given order, this Bulletin, vol. 29 (1923), pp. 407-414.

9. Hollcroft, T. R., Maximal cuspidal curves, Annals of Mathematics, (2), vol. 26 (1924), pp. 37-46.

10. Hollcroft, T. R., On the reality of singularities of plane curves, Mathematische Annalen, vol. 97 (1927), pp. 775-787.

11. Hollcroft, T. R., Invariants associated with singularities of algebraic curves, Acta Mathematica, vol. 56 (1930), pp. 261-272.

12. Klein, F., Eine neue Relation zwischen den Singularitäten einer algebraischen Kurve, Mathematische Annalen, vol. 10 (1876), pp. 199-209.

13. Lefschetz, S., On the existence of loci with given singularities, Transactions of this Society, vol. 14 (1913), pp. 23-41.

14. del Pezzo, P., Equazione d'una curva del quinto ordine dotata di cinque cuspidi, Rendiconti, Accademia delle Scienze Fisiche di Napoli, (2), vol. 3 (1889), pp. 46-49.

15. Plücker, J., Theorie der algebraischen Curven, Bonn, 1839.

16. Segre, B., Esistenza e dimensione di sistemi continui di curve piane algebriche con dati caratteri, Lincei Rendiconti, (6), vol. 10 (1929), pp. 31-38.

17. Segre, B., Esistenza di sistemi continui distinti di curve piane algebriche con dati numeri plueckeriani, Lincei Rendiconti, (6), vol. 10 (1929), pp. 557-560.

18. Segre, B., Sulla caratterizzazione delle curve di diramazione de piani multipli generali, Memorie, Reale Academia d'Italia, Classe di Scienze Fisiche, Matematiche e Naturali, vol. 1 (1930), pp. 5-31.

19. Severi, F., Vorlesungen über algebraische Geometrie, Severi-Löffler, Berlin, 1921, Anhang F, pp. 307-353.

20. Snyder, V., Construction of plane curves of given order and genus, having distinct double points, this Bulletin, vol. 15 (1908), pp. 1-4.

21. Zariski, O., On the problem of existence of algebraic functions of two variables possessing a given branch curve, American Journal of Mathematics, vol. 51 (1929), pp. 305-328. 
22. Zariski, O., On the linear connection index of the algebraic surfaces $z^{n}=f(x, y)$, Proceedings of the National Academy of Sciences, vol. 15 (1929), pp. 494-501.

23. Zariski, O., On the non-existence of curves of order 8 with 16 cusps, American Journal of Mathematics, vol. 53 (1931), pp. 309-318.

24. Zariski, O., On the irregularity of cyclic multiple planes, Annals of Mathematics, (2), vol. 32 (1931), pp. 485-511.

25. Zariski, O., Algebraic Surfaces, Ergebnisse der Mathematik, vol. 3 (1935), pp. 160-181.

Wells College

\section{JENSEN'S INEQUALITY*}

BY E. J. MCSHANE

The simplest form of Jensen's inequality is that if $\phi(x)$ is a convex function, and $m$ is the arithmetic mean of $x_{1}, \cdots, x_{n}$, then the mean of the numbers $\phi\left(x_{n}\right)$ is not less than $\phi(m)$. This inequality can be generalized in several different ways. The function $\phi(x)$ can be replaced by a convex function of several variables, and the arithmetic mean can be replaced by any one of several other means, as has been shown in various proofs. Since the inequality is of considerable utility, it seems worth while to have it established in a form which is general enough to cover a wide assortment of applications.

The proofs will rest on two well known properties of convex sets. $\dagger$ If $K$ is closed and convex and a point $p$ is not in $K$, then $p$ can be separated from $K$ by a hyperplane. If $K$ is closed and convex and $p$ is a boundary point of $K$, there is a hyperplane of support of $K$ passing through $p$.

1. The Inequality in Geometric and in Analytic Form. It will be convenient in the following proofs to use these symbols and definitions:

$R_{n}$ is $n$-dimensional Euclidean space. Its points will be denoted by $\left(z_{1}, \cdots, z_{n}\right)$ or by $z$. Linear functions $\sum a_{i} z_{i}$ or $R_{n}$ will be symbolized by $l(z)$.

\footnotetext{
* Presented to the Society, December 31, 1936.

$\dagger$ A set is convex if for every pair $P, Q$ of points of the set the line segment $P Q$ is contained in the set.
} 